\title{
Combined heat and mass transfer in mixed convection through a horizontal tube
}

\author{
Aicha Bouhezza $^{1}$, Omar Kholai $^{2 *}$, Saadoun Boudebous $^{3}$, Zoubir Nemouchi ${ }^{4}$ \\ ${ }^{1}$ Département de Technologie, Faculté de Technologie, Université 20 Août 1955-Skikda,, Skikda 21000, Algeria \\ ${ }^{2}$ Département de Génie des Transports, Université des Frères Mentouri, Constantine 25000, Algeria \\ ${ }^{3}$ Département de Génie Mécanique, Faculté des Sciences et des Sciences Appliquées, Université Larbi Ben M'hidi Oum El \\ Bouaghi, Oum El Bouaghi 04000, Algeria \\ ${ }^{4}$ LEAP, Département de Génie Mécanique, Université Frères Mentouri Constantine 1, Constantine 25000, Algeria
}

Corresponding Author Email: kholai.omar@gmail.com

https://doi.org/10.18280/ijht.360110

Received: 13 October 2017

Accepted: 24 November 2017

\section{Keywords:}

heat transfer, mass transfer, mixed convection, schmidt number, horizontal tube $(3 D)$, elliptic equations

\begin{abstract}
Laminar mixed convection heat and mass transfer in horizontal circular tube with uniform heat and mass fluxes at the walls on part of its length have been investigated numerically using a three - dimensional elliptic equations model. The effect of the Schmidt number and the buoyancy ratio $\mathrm{N}$, on the development of the hydrodynamic, thermal and solutal fields and the axial evolution of the Nusselt and the Sherwood numbers are presented. These results showed that the Schmidt number has the highest effect on the flow rather than the Nusselt and Sherwood numbers. The Nusselt number and Sherwood number increase for positive $\mathrm{N}$ and decrease when $\mathrm{N}$ is negative.
\end{abstract}

\section{INTRODUCTION}

The fluid flow with heat and mass transfer is a subject which continues to cause much interest within the scientific community because of their many industrial applications such as the compact heat exchangers, cooling of the electronic components, chemical distillation, solar energy collectors, and others.

Generally, these flows are characterized by the presence of the temperature and concentration gradients which generate thermal and solutal buoyancy forces.

Mixed convection in ducts due to the effect of thermal buoyancy was studied numerically and experimentally for both aiding (the flow is in the same direction as the buoyancy force) and opposing (the flow is in the opposite direction to the buoyancy force) flows by various authors, including Choi and Choi (1994), Joye (1996), Choudhury and Patankar (1988), Zghal et al. (2001), Maré et al. (2006), and Mohammed (2008). However, the mixed convection due to the combined effects of thermal and solutal buoyancies has received less attention. Chang et al. (1986) investigated numerically the natural convection flows in a vertical, open tube resulting from combined buoyancy effects of thermal and mass diffusion. Results were presented for an air-water system. The effects of tube length as well as system temperatures and pressures on the heat and mass transfer in the flow are examined in great detail. The results show the important role that the liquid film plays under the situations of buoyancy-aiding and opposing flows. The developing laminar mixed convection with heat and mass transfer in inclined rectangular ducts was studied numerically by Yan (1995). Typical developments of velocity, temperature, and concentration profiles were shown. The local friction factor, Nusselt number, and Sherwood number were presented for the transported species of interest in air $(\mathrm{Pr}=0.7)$ over a Schmidt number range of 0.2-2, and the buoyancy ratio $N$ was varied between -0.8 and 2.0. Lee (1999) investigated numerically the laminar natural convection heat and mass transfer in open vertical rectangular ducts with uniform temperature and uniform concentration or uniform heat flux and uniform mass flux at the wall. The governing equations are solved by the vorticity-velocity method for threedimensional parabolic flow. Results were obtained for a fluid with $\operatorname{Pr}=0.7$ and $S c=0.2,0.6$ and 1.3, and the buoyancy ratio $N=0,1$ and 2 (the solutal buoyancy force acts in the same direction as the thermal buoyancy force). The author proposed correlations for the volume rate, and average Nusselt and Sherwood numbers for both boundary conditions. Orfi and Galanis (2005) analyzed the effect of the Lewis number on laminar mixed convection heat and mass transfer in a horizontal tube with uniform heat and uniform concentration at the wall. Results were obtained for $\operatorname{Pr}=0.7$, the buoyancy force from species diffusion assists the thermal buoyancy force and values of the Lewis number ranging from 0.2 to 5 . Recently, the present authors (2013) studied the effects of thermal and solutal buoyancy forces and inclination angle on the development of laminar mixed convection in a circular inclined duct with uniform heat flux and uniform concentration at the wall. These results were limited to the case when the solutal buoyancy force acts in the same direction as the thermal buoyancy force, and $S c=0.6$. Therefore, the present work is devoted to study the effect of the Schmidt number $S c$ and the buoyancy ratio $\mathrm{N}$ on the development of laminar mixed convection heat and mass transfer in horizontal circular tube with uniform heat and mass fluxes at the wall.

\section{PHYSICAL MODEL AND MATHEMATICAL FORMULATION}

The geometry of the problem under consideration is shown in Fig. 1. The fluid is a mixture of two non-reacting gases (large amounts of species A and small amounts of species B). 
The fluid enters into a horizontal circular duct of radius $R$ at $z^{*}$ $=-L_{1}$ with a uniform velocity $V_{0}{ }^{*}$ and a constant inlet temperature $T_{0}{ }^{*}$ and concentration $C_{0}{ }^{*}$ (mass of B per total mass). A uniform heat flux $q_{w}$ and uniform mass flux $m_{w}$ are imposed at the wall from $z^{*}=0$ to $z^{*}=L_{2}$ while the rest of wall is adiabatic and impermeable. The flow is considered to be unsteady, laminar, and three-dimensional. The following assumptions are adopted:

1) The fluid is Newtonian and incompressible; all its thermo-physical properties are considered constant except for the density in the buoyancy terms which varies linearly both with temperature and concentration;

$$
\rho=\rho_{0}\left[1-\beta_{T}\left(T^{*}-T_{0}^{*}\right)-\beta_{C}\left(C^{*}-C_{0}^{*}\right)\right]
$$

Here $\beta_{T}$ and $\beta_{C}$ are the thermal and concentration expansion coefficients, respectively;

2) The heat transfer by radiation in the walls is negligible because the temperature range considered is small, (Gebhart and Pera, 1971; Yan, 1995; Orfi and Galanis, 2005).

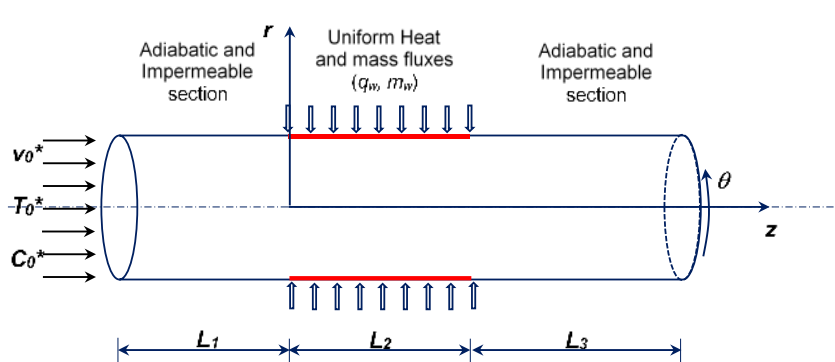

Figure 1. Geometrical configuration of the studied case

3) The species diffusion due to the temperature gradient (Soret effect) and the heat diffusion due to the concentration gradient (Dufour effect) are considered negligible. These secondary effects are not taken into account since the temperature and concentration gradients considered in the present work are low (Gebhart and Pera, 1971; Yan, 1995; Orfi and Galanis, 2005).

4) The interfacial velocity at the tube walls as a result of species diffusion process is neglected. This is because the model is based on species diffusion processes with very low concentration levels (Yan, 1995; Orfi and Galanis, 2005).

5) Viscous dissipation, enthalpy interdiffusion, and pressure work are negligible.

Dimensionless variables are obtained by taking the diameter $(2 R)$ and the axial velocity $\left(V_{0}{ }^{*}\right)$ as the characteristic length and velocity, respectively. Dimensionless time, pressure, temperature, and concentration are given respectively by the following expressions:

$$
\begin{aligned}
& t=\frac{t^{*} V_{0}^{*}}{2 R}, \\
& P=\frac{P^{*}+\rho_{0} g\left(r^{*} \cos \theta\right)}{\rho_{0} V_{0}^{* 2}} \\
& T=\frac{\left(T^{*}-T_{0}^{*}\right)}{q_{w}(2 R / k)} \\
& C=\frac{\left(C^{*}-C_{0}^{*}\right)}{m_{w}(2 R / D)}
\end{aligned}
$$

It should be noted that all the results presented in this work pertain to the steady flow solution. Unsteady-state effects were not explored. The use of transient terms in the equations is advantageous from the point of view of the numerical method of solution.

The mass, momentum, energy, and concentration equations in cylindrical coordinates $(r, \theta, z)$ can then be written in the following nondimensional form:

$$
\begin{aligned}
& \frac{1}{r} \frac{\partial(r u)}{\partial r}+\frac{1}{r} \frac{\partial w}{\partial \theta}+\frac{\partial v}{\partial z}=0 \\
& \frac{\partial u}{\partial t}+u \frac{\partial u}{\partial r}+\frac{w}{r} \frac{\partial u}{\partial \theta}+v \frac{\partial u}{\partial z}-\frac{w^{2}}{r}=-\frac{\partial P}{\partial r}+\frac{1}{\operatorname{Re}}\left[\frac{\partial}{\partial r}\left(\frac{1}{r} \frac{\partial(r u)}{\partial r}\right)+\frac{1}{r^{2}} \frac{\partial^{2} u}{\partial \theta^{2}}-\frac{2}{r^{2}} \frac{\partial w}{\partial \theta}+\frac{\partial^{2} u}{\partial z^{2}}\right] \\
& +\left(\frac{G r_{T}}{\operatorname{Re}^{2}} T+\frac{G r_{C}}{\operatorname{Re}^{2}} C\right) \cos \theta \\
& \frac{\partial w}{\partial t}+u \frac{\partial w}{\partial r}+\frac{w}{r} \frac{\partial w}{\partial \theta}+v \frac{\partial w}{\partial z}-\frac{u w}{r}=-\frac{1}{r} \frac{\partial P}{\partial \theta}+\frac{1}{\operatorname{Re}}\left[\frac{\partial}{\partial r}\left(\frac{1}{r} \frac{\partial(r w)}{\partial r}\right)+\frac{1}{r^{2}} \frac{\partial^{2} w}{\partial \theta^{2}}-\frac{2}{r^{2}} \frac{\partial u}{\partial \theta}+\frac{\partial^{2} w}{\partial z^{2}}\right](5) \\
& -\left(\frac{G r_{T}}{\operatorname{Re}^{2}} T+\frac{G r_{C}}{\operatorname{Re}^{2}} C\right) \sin \theta \\
& \frac{\partial v}{\partial t}+u \frac{\partial v}{\partial r}+\frac{w}{r} \frac{\partial v}{\partial \theta}+v \frac{\partial v}{\partial z}=-\frac{\partial P}{\partial z}+\frac{1}{\operatorname{Re}}\left[\frac{1}{r} \frac{\partial}{\partial r}\left(r \frac{\partial v}{\partial r}\right)+\frac{1}{r^{2}} \frac{\partial^{2} v}{\partial \theta^{2}}+\frac{\partial^{2} v}{\partial z^{2}}\right] \\
& \frac{\partial T}{\partial t}+u \frac{\partial T}{\partial r}+\frac{w}{r} \frac{\partial T}{\partial \theta}+v \frac{\partial T}{\partial z}=\frac{1}{\operatorname{Re} \operatorname{Pr}}\left[\frac{1}{r} \frac{\partial}{\partial r}\left(r \frac{\partial T}{\partial r}\right)+\frac{1}{r^{2}} \frac{\partial^{2} T}{\partial \theta^{2}}+\frac{\partial^{2} T}{\partial z^{2}}\right] \\
& \frac{\partial C}{\partial t}+u \frac{\partial C}{\partial r}+\frac{w}{r} \frac{\partial C}{\partial \theta}+v \frac{\partial C}{\partial z}=\frac{1}{\operatorname{Re} S c}\left[\frac{1}{r} \frac{\partial}{\partial r}\left(r \frac{\partial C}{\partial r}\right)+\frac{1}{r^{2}} \frac{\partial^{2} C}{\partial \theta^{2}}+\frac{\partial^{2} C}{\partial z^{2}}\right]
\end{aligned}
$$

The dimensionless numbers characterizing the phenomenon are: the Prandtl number $\operatorname{Pr}=v / a$, where $a$ is the thermal diffusivity of the fluid and $v$ is the kinematic viscosity of the fluid, the Schmidt number $S c=v / D$, where $D$ is the mass diffusivity, the Reynolds number $R e=V_{0} * 2 R / v$, the thermal Richardson number $R i_{\mathrm{T}}=G r_{\mathrm{T}} / \mathrm{Re}^{2}$, where the thermal Grashof number $G r_{\mathrm{T}}=g \beta_{\mathrm{T}} q_{w}(2 R)^{4} / v^{2} k$, and the solutal Richardson number $R i_{\mathrm{C}}=G r_{\mathrm{C}} / \mathrm{Re}^{2}$, where the solutal Grashof number $G r_{\mathrm{C}}$ $=g \beta_{\mathrm{C}} m_{w}(2 R)^{4} / v^{2} D$. The buoyancy ratio $N=R i_{\mathrm{C}} / R i_{\mathrm{T}}$ represents the ratio between solutal and thermal buoyancy forces. The buoyancy force from species diffusion assists the thermal buoyancy force when $N>0$, whereas it opposes the thermal buoyancy force when $N<0$. When $N=0$, there is no species diffusion and the buoyancy force is due to the thermal diffusion only.

The system of preceding equations is elliptic in the three directions. It is subjected to the following boundary conditions: at the tube entrance

$\left(z^{*}=-L_{1}\right): u=0, w=0, v=1, T=0$ and $C=0$

at the tube outlet

$\left(z^{*}=L_{2}+L_{3}\right): \frac{\partial u}{\partial z}=0, \frac{\partial w}{\partial z}=0, \frac{\partial v}{\partial z}=0, \frac{\partial T}{\partial z}=0$ and $\frac{\partial C}{\partial z}=0(9 \mathrm{~b})$ at the wall $(r=0.5): u=0, w=0, v=0$,

$\frac{\partial T}{\partial r}=1$ and $\frac{\partial C}{\partial z}=1$,

for $0 \leq z^{*} \leq L_{2}$ 
$\frac{\partial T}{\partial r}=0$ and $\frac{\partial C}{\partial z}=0$ elsewhere

at the tube axis

$(r=0): \frac{\partial}{\partial r}\left(\frac{\partial u}{\partial r}\right)=\frac{\partial}{\partial r}\left(\frac{\partial w}{\partial r}\right)=\frac{\partial}{\partial r}\left(\frac{\partial v}{\partial r}\right)=\frac{\partial}{\partial r}\left(\frac{\partial T}{\partial r}\right)=\frac{\partial}{\partial r}\left(\frac{\partial C}{\partial r}\right)=0,(9 \mathrm{e})$

at $(\theta=0)$ and $(\theta=2 \pi)$ :

$u(r, 0, z, t)=u(r, 2 \pi, z, t), w(r, 0, z, t)=w(r, 2 \pi, z, t)$,

$v(r, 0, z, t)=v(r, 2 \pi, z, t), T(r, 0, z, t)=T(r, 2 \pi, z, t)$ and

$C(r, 0, z, t)=C(r, 2 \pi, z, t)$.

The local and circumferentially average Nusselt numbers are given by:

$N u_{\theta}=\frac{1}{T_{\theta}-T_{b}}, N u_{z}=\frac{1}{\overline{T_{w}}-T_{b}}$

where:

$$
\overline{T_{w}}=\frac{1}{2 \pi} \int_{0}^{2 \pi} T_{w} d \theta \text { and } T_{b}=\frac{\int_{0}^{0.5} \int_{0}^{2 \pi} v \cdot T \cdot r \cdot d \theta \cdot d r}{\int_{0}^{0.52 \pi} \int_{0}^{2 \pi} v \cdot r \cdot d \theta \cdot d r},
$$

The local and circumferentially average Sherwood numbers are defined as:

$S h_{\theta}=\frac{1}{C_{\theta}-C_{b}}, S h_{z}=\frac{1}{\overline{C_{w}}-C_{b}}$

where:

$\overline{\mathrm{C}_{w}}=\frac{1}{2 \pi} \int_{0}^{2 \pi} \mathrm{C}_{w} d \theta \quad$ and $\quad \mathrm{C}_{b}=\frac{\int_{0}^{0.5} \int_{0}^{2 \pi} v \mathrm{C} r d \theta d r}{\int_{0}^{0.5} \int_{0}^{2 \pi} v r d \theta d r}$

\section{NUMERICAL METHODS}

In the present work, the discretization of the coupled, nonlinear, elliptic partial differential equations was based on the finite volume approach. For the convective and diffusive terms a power-law scheme was used while the SIMPLER procedure (Patankar, 1980) was introduced for the velocitypressure coupling. The fully implicit scheme was used to discretize the temporal terms. The resulting discretized equations were solved iteratively, using the Tri-Diagonal Matrix Algorithm. The time increment is $\Delta t=10^{-4}$. The numerical mesh generated is uniform in the circumferential direction and non-uniform in the axial and radial directions so as to capture the relatively important variation in the thermal, concentration, and hydrodynamic fields, particularly in the heated zone. The convergence of the solution was considered reached when the maximum relative change of all the variables

( $u, w, T$ and $C$ ) between two successive time steps was lower than $10^{-6}$.

To ensure the independence of results for the grid used, three grids were tested (Bouhezza et al. 2013). In addition, the numerical model was validated by comparing our results with experimental and numerical published results, considering the configurations reported in Orfi and Galanis (2002), and Kays and Crawford (1993), and Kakac et al. (1987).

\section{RESULTS AND DISCUSSION}

Numerical results were obtained for species diffusion in air $(\operatorname{Pr}=0.7)$ with Schmidt numbers ranging from 0.22 to 2.01 . This covers diffusion into air of hydrogen $(S c=0.22)$, water vapor $(S c=0.6)$, ethanol vapor $(S c=1.3)$ and benzene vapor $(S c=2.01)$ (Yan, 1995). The values of the buoyancy ratio $N$ was varied from -1.0 to 2.0. For each case the Reynolds number and thermal Richardson number are fixed at 400 and 0.62 , respectively. The results presented relate to the middle part of the tube where a uniform heat and mass fluxes value are prescribed.

\subsection{Effect of the Schmidt number}
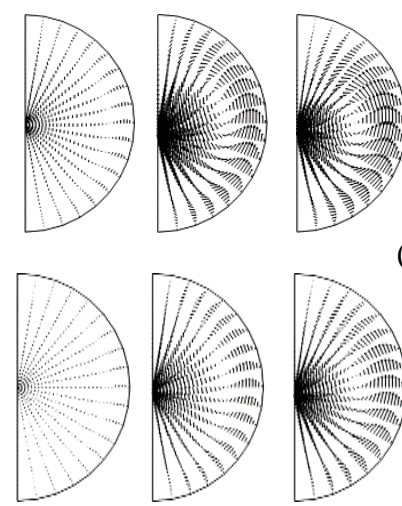

(a)

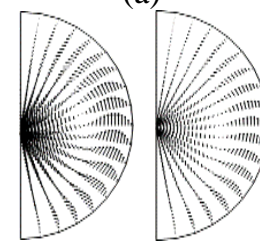

(b)

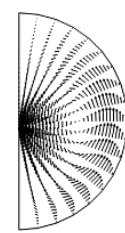

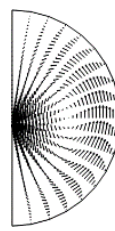
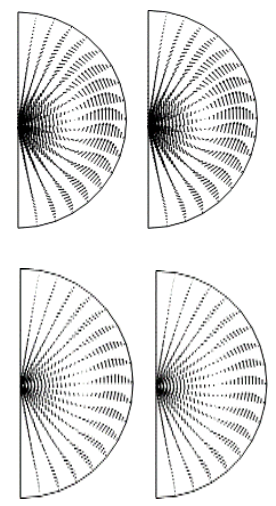

Figure 2. Projected vector plots (A, B, C, D, E and F) showing the secondary flow in a-tube: (a) Schmidt number $S c$ $=0.22$; (b) Schmidt number $S c=2.01$

The effects of Schmidt number on the flow, temperature and concentration fields are presented and discussed.

Figures $2 \mathrm{a}$ and $2 \mathrm{~b}$ shows the buoyancy induced secondary flow distribution, at six different cross sections A, B, C, D, E and $\mathrm{F}$, of the tube situated at $z=0.6053,3.2286,7.4664$, 14.5291, 20.3811 and 24.8206, respectively. For two cases $S c$ $=0.22$ and $S c=2.01$ at the first axial position, $z=0.6053$, it is clear that the secondary flow is already affected by the thermal and solutal buoyancy forces. The magnitudes of the velocity vectors are relatively larger when $S c=0.22$. As the fluid moves downstream, the buoyancy forces are an important effect for the two cases. On the other hand, the strength of the secondary flow increases and becomes most intense at $z=$ 7.4664 (section C) for $S c=0.22$ and $S c=2.01$. The ascending part of the vortices is greater for $S c=0.22$. Farther downstream, at $z=24.8206$ the effects of thermal and solutal buoyancy are never disappeared since the conditions of heat and mass fluxes, and after this part the flow becomes fully developed.

The effects of the Schmidt number on the development of the axial velocity profiles along the vertical diameter of symmetry are presented in Figs. 3. Close to the entrance of the 
middle part of the tube (position A, at $z=0.6053$ Fig 3 (a)) the velocity profiles are uniform in the central part and symmetrical with respect to the tube axis and these profiles for all cases are quite close. But as the fluid goes downstream Fig 3 (b) (c), this symmetry is lost, and the fluid is accelerated and the maximum axial velocity move toward the lower half of the tube. So, the axial velocity is reduced with the reduction in the Schmidt number.

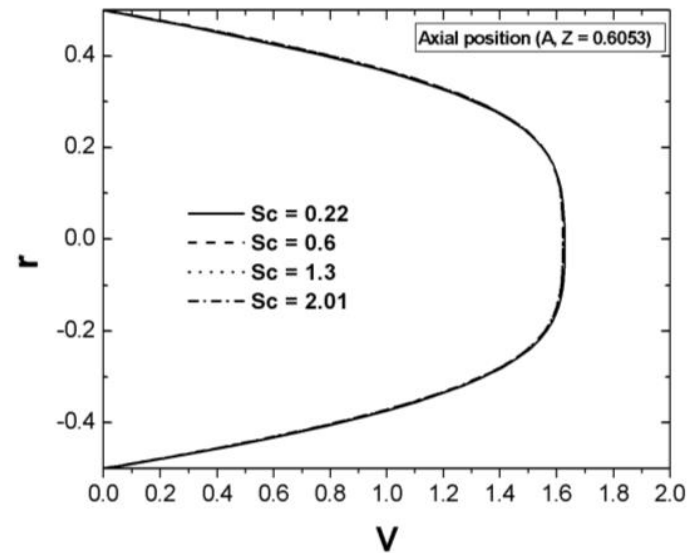

(a)

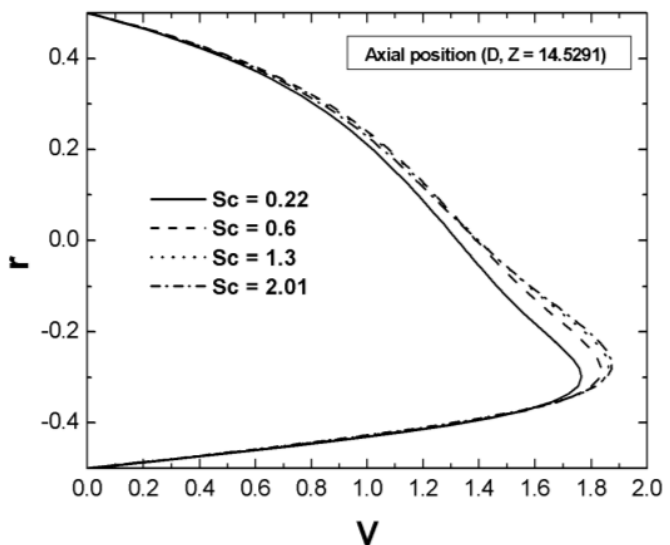

(b)

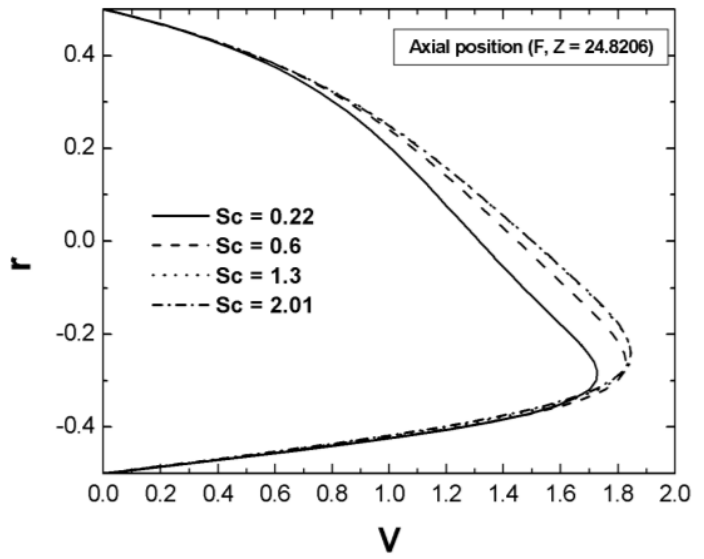

(c)

Figure 3. Effect of Schmidt number $S c$ on the evolution of axial velocity at different positions A, D and F

The isoconcentrations at the same axial positions are presented in Figs. 4a, b. In both cases, near the entrance of the middle part of the tube $(z=0.6053$, position A), the isoconcentrations are nearly circular, due to the weak secondary flow. Further downstream, at $z=7.4664$ the isoconcentrations contours are distorted. The distortion of these contours is more important in the upper half of the tube because of the high intensity of the secondary flow (Figs. 2). In the lower half of the tube these contours are almost circular. It is noted that the distortion of these contours is much more important when $S c=2.01$.
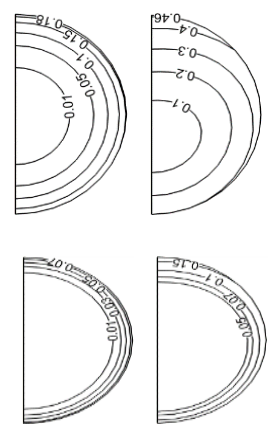

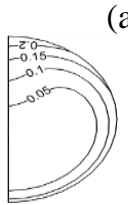

(b)

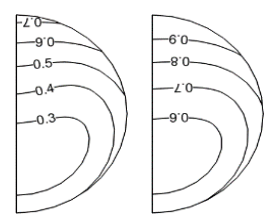

(a)

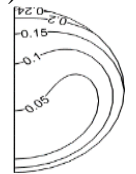

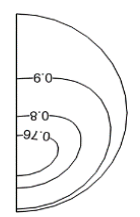

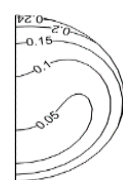

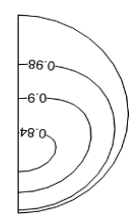

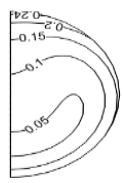

Figure 4. Contours of the isoconcentration in a horizontal tube: (a) Schmidt number $S c=0.22$; (b) Schmidt number $S c$ $=2.01$

The profiles of the concentration for various values of the Schmidt number and different positions of the tube are shown in Figs. 5a, 5b, 5c. From these figures, it is clear that, close to the entrance of the middle part of the tube, the concentration profiles are symmetrical with the axis of the tube for the four cases considered and also, the concentration do not vary according to $\theta$. But as the fluid goes downstream, the concentration profiles lose their symmetry, and the minimal values of concentration moves towards the lower half of the tube. The concentration varies according to $\theta$, its maximum value always corresponds to the top of the tube $(\theta=0)$, while its minimal concentration is at the bottom of the tube $(\theta=\pi)$. The gradients of concentration decrease when the Schmidt number increases. Finally, it is noted that the concentration field develops very quickly compared to the temperature field when $S c<\operatorname{Pr}$, and when $S c>\mathrm{Pr}$, the concentration field develops slower than the thermal field.

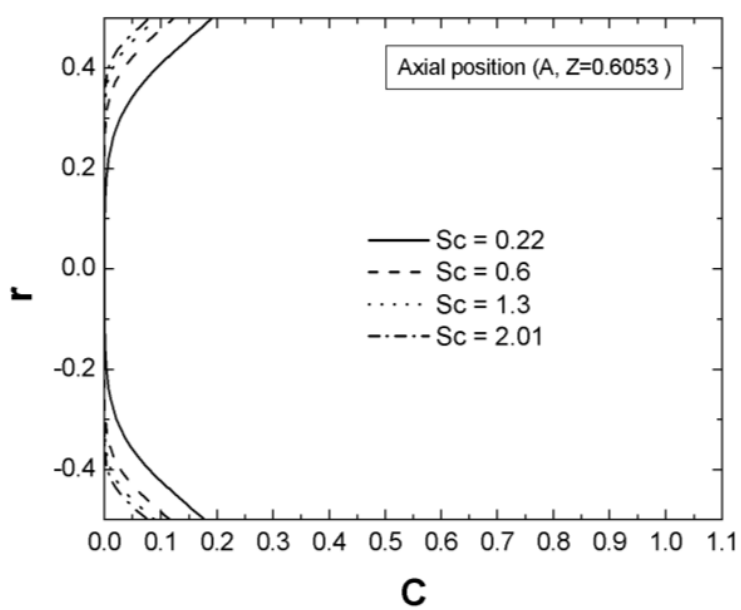

(a) 


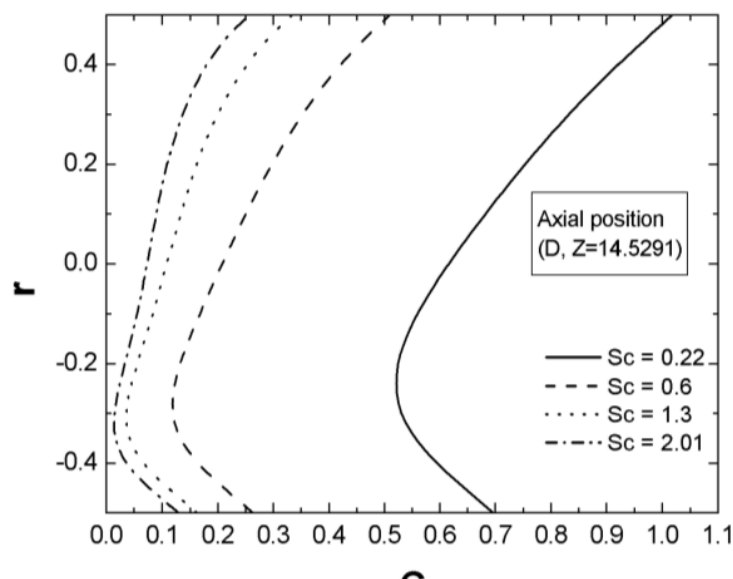

C

(b)

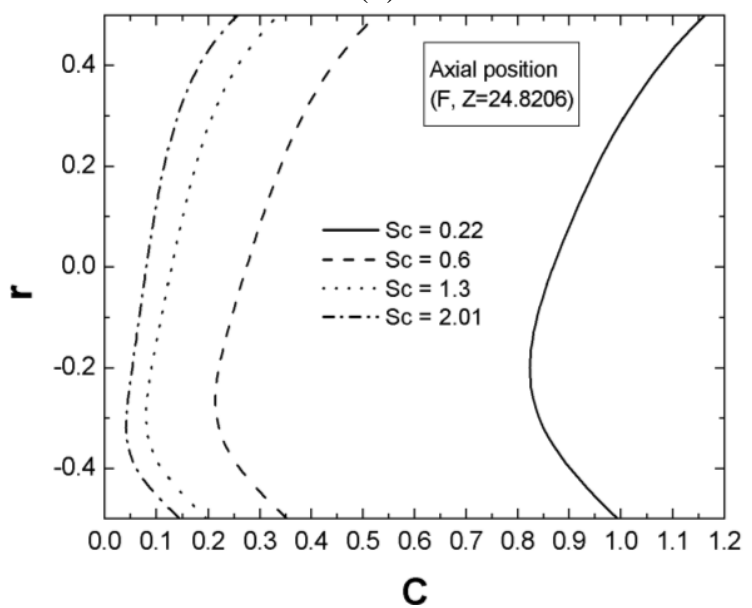

(c)

Figure 5. Effect of Schmidt number Sc on the evolution of the concentration profiles at different positions A, D and F

Figure 6 illustrates the effect of the Schmidt number on the axial evolution of the circumferentially averaged Nusselt number. The pure forced convection curve, has been obtained with $S c=0.6$. It can be seen from this figure that the Schmidt number effect is nonexistent near the inlet of the middle part of the tube (where the effects of thermal and solutal buoyancy forces does not exist) and the $N u_{Z}$ decreases in close agreement with the corresponding evolution for forced convection. As the buoyancy effects become important (at about $z=3.5$ ), the $N u_{Z}$ curves for solutal and thermal mixed convection rise above the forced convection curve. The $S c=0.22$ curve is markedly different compared to the $S c=0.6, S c=1.3$ and $\mathrm{Sc}=2.01$ curves. In addition, the vigorous secondary flow result causes an increase in the heat transfer. The circumferentially averaged Nusselt number results show a diminishing influence of increasing the Schmidt number from 0.6 to 2.01 .

The effect of Schmidt number on the axial evolution of the circumferentially averaged Sherwood number is presented in Figure 7. It is clearly seen that the $S h_{Z}$ curves are quite different. Near the entrance of the middle part of the tube, the values of $S h_{Z}$ are smaller than the corresponding values for forced convection when $S c<\mathrm{Pr}$ and the values of $S h_{Z}$ are higher than the corresponding values for forced convection when $S c>\operatorname{Pr}$. Further downstream, for all cases, the $S h_{Z}$ are larger than the corresponding values for forced convection. On the other hand, the Sherwood number increases as the Schmidt number increase. This is explained by the reduction of the concentration boundary layer thickness compared to the dynamic boundary layer thickness with the increase in the Schmidt number. Therefore, the rate of mass transfer increases (the Sherwood number increases).

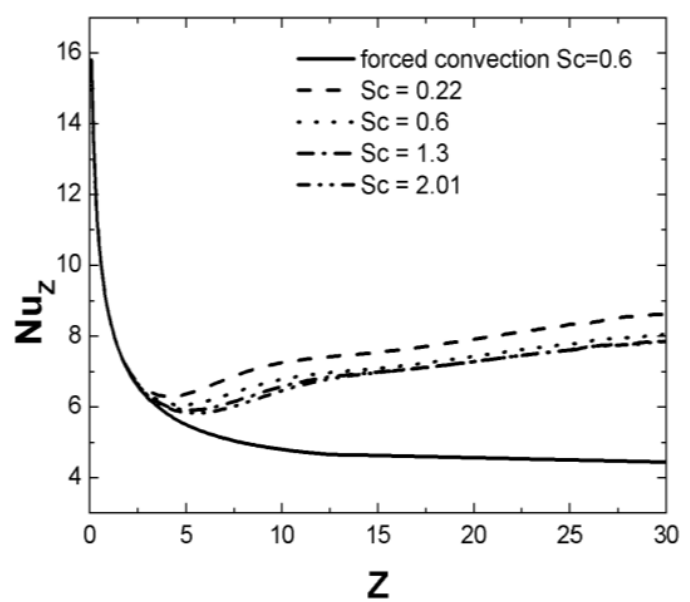

Figure 6. Effect of Schmidt number $S c$ on the circumferentially averaged Nusselt number in a horizontal tube

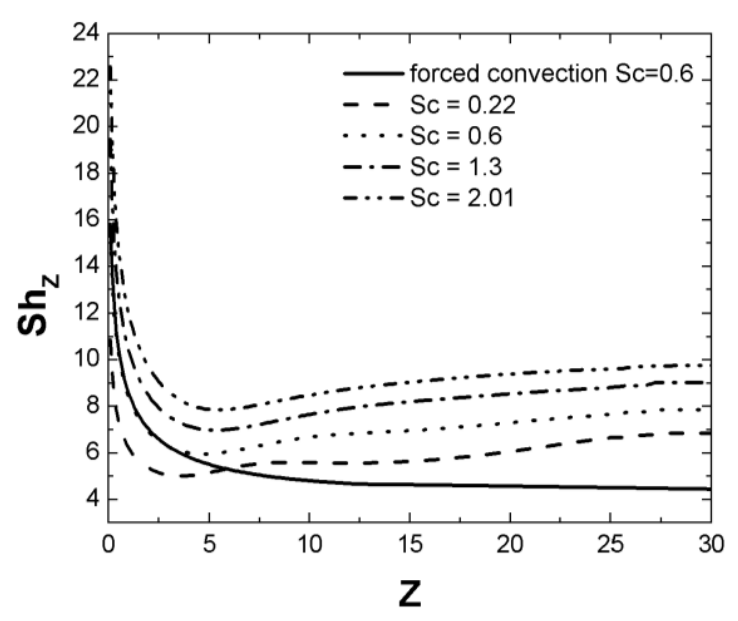

Figure 7. Effect of Schmidt number $S c$ on the circumferentially averaged Sherwood number in a horizontal tube

\subsection{Effect of the buoyancy ratio $\mathrm{N}$}

The axial evolution of the secondary flows induced by the thermal and mass buoyancy forces for different buoyancy ratios $N$ are compared in Figs. 8a, b, c, d. Near the entrance of the middle part of the tube (section A), it is observed that the appearance of the secondary flow for case $N=2$ (Figure 8a). At the same axial location, for the other cases (Figs. 8b, c, d), the secondary flow is negligible. As the fluid moves downstream, for the three cases $(N=2,1,0)$, one notes the appearance of the secondary flow. These secondary movements become more important at about $z=7.4664$, and thereafter decrease tending towards different fully developed states. By comparing the results of these cases, one notes that the increase in the buoyancy ratio $N$ amplifies the intensity of the secondary flow and the reduction in this parameter reduces these secondary movements. For $N=-1$, these secondary movements disappear along the tube. This indicates that the solutal buoyancy force acts in the opposite direction of the thermal buoyancy force, and whose total contribution is null, 
thus, the mixed convection behaves then like a forced convection.

Figures 9a, b, c illustrates the effect of the buoyancy ratios $N$ on the development of the axial velocity profiles along the symmetry diameter. Near to the entrance of the middle part of the tube $(z=0.6053$, section A), the axial velocity profiles are uniform in the central zone and symmetrical with respect to the tube axis and essentially identical for the all cases. But as the fluid goes downstream, this symmetry is lost, and the profiles are distorted due to buoyancy effects, and the nature of distortion depends on the buoyancy ratio $N$. The secondary flow induced by the buoyancy forces has a direct influence on axial velocity, and the maximum axial velocity moves towards the lower half of the tube, but for $N=-1$ the axial velocity profile is always symmetrical. At the position $z=24.8206$ (far from the entrance), by comparing maximum velocities for all the cases, one deduces that the maximum value of the axial velocity for the case $N=-1$ is definitely higher than that for other cases.

The effect of the buoyancy ratio $N$ on the axial evolution of the local Nusselt and Sherwood numbers is illustrated in Figs. 10 and 11 . These figures show clearly that the curves of the $N u_{\theta}$ and $S h_{\theta}$ numbers are symmetrical compared to the vertical diameter of the tube $(\theta=\pi)$ along the tube. Close to the entrance of the middle part of the tube these curves are independent of $\theta$, and primarily identical for all the cases, i.e. the influence of the buoyancy ratio is negligible. But as the fluid goes downstream, the shape of the curves of $N u_{\theta}$ and $S h_{\theta}$ is influenced by ratio $N$. For cases $N \neq-1$, one notes that the values of these numbers increase when $\mathrm{z}$ increases and thereafter decrease tending towards different fully developed states with the increase in $z$. The maximum values of $N u_{\theta}$ and $S h_{\theta}$ are located at the bottom of the tube $(\theta=\pi)$, while its minimal values are located at the top of the tube $(\theta=0)$. For the case $N=-1$ these numbers do not vary according to the angular direction along the tube because of the absence of the secondary movements, as noticed in the Figure 8d.
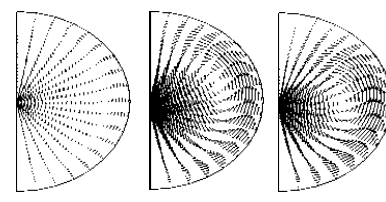

(a)

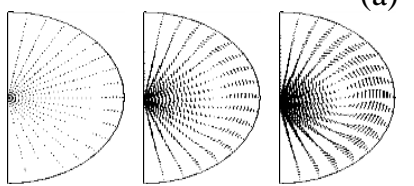

(b)

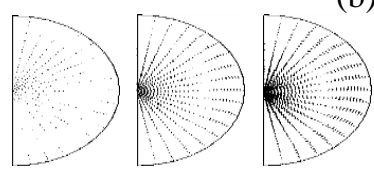

(c)

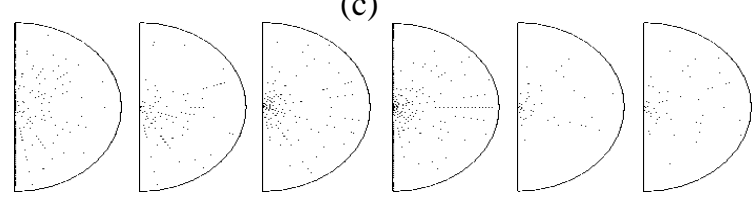

(d)

Figure 8. Projected vector plots showing the secondary flow: (a) buoyancy ratio $N=2$; (b) buoyancy ratio $N=0$; (c) buoyancy ratio $N=-0.5$; (d) buoyancy ratio $N=-1$

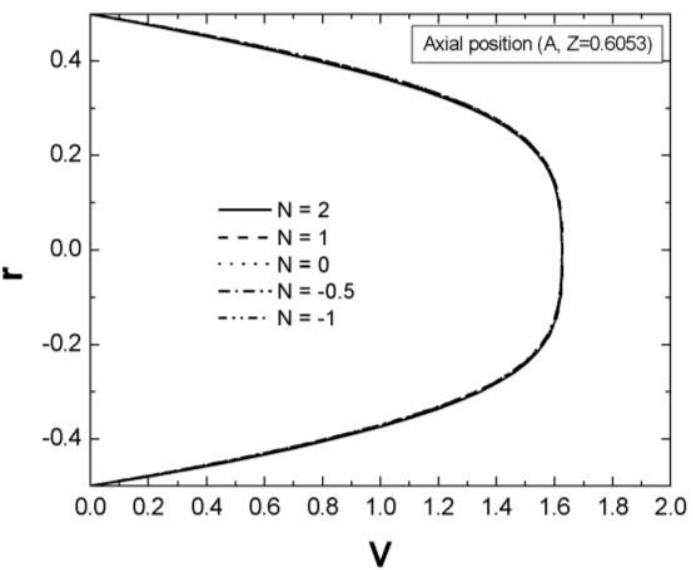

(a)

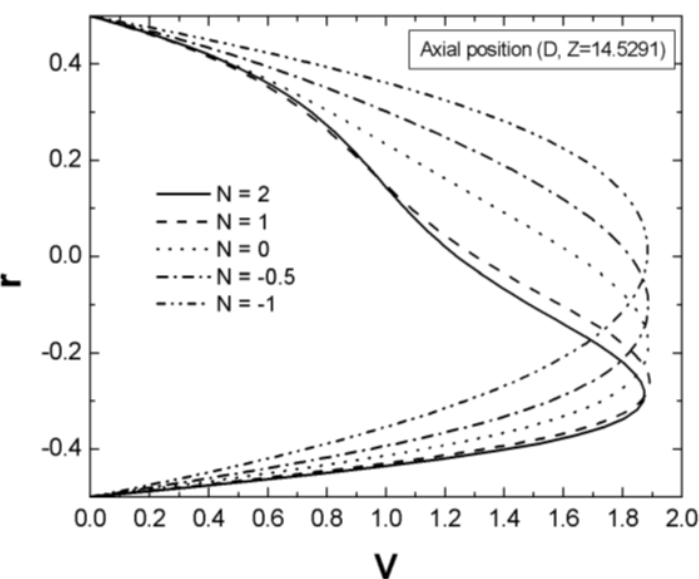

(b)

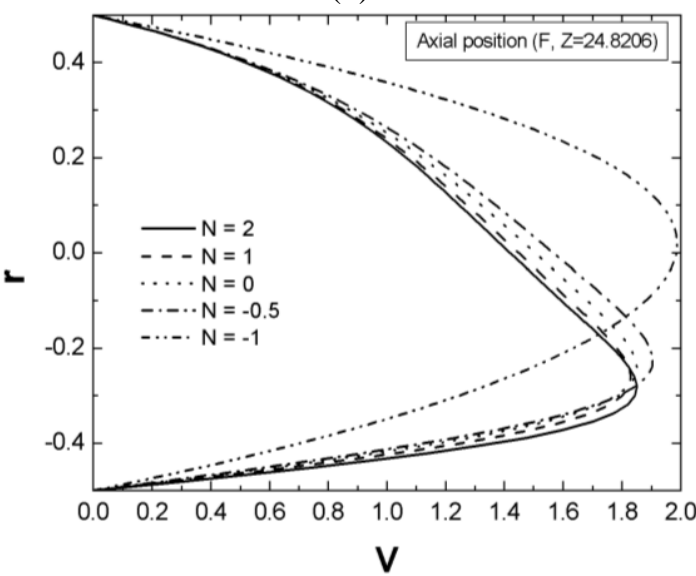

(c)

Figure 9. Effect of buoyancy ratio $N$ on the evolution of axial velocity profiles at different positions $\mathrm{A}, \mathrm{D}$ and $\mathrm{F}$

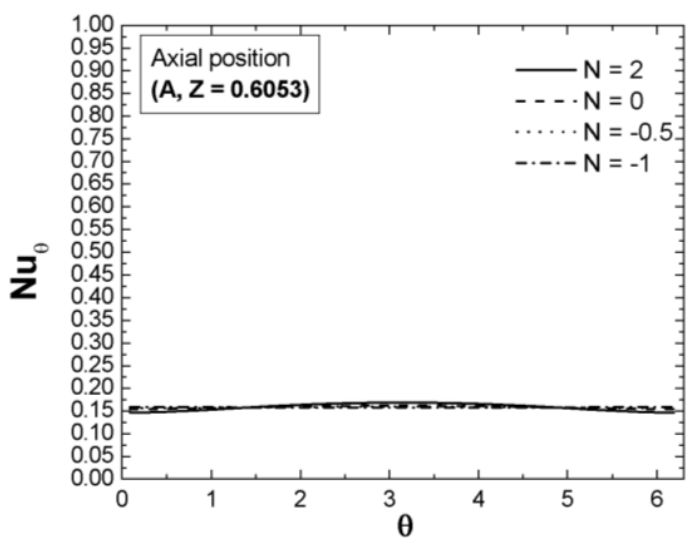

(a) 


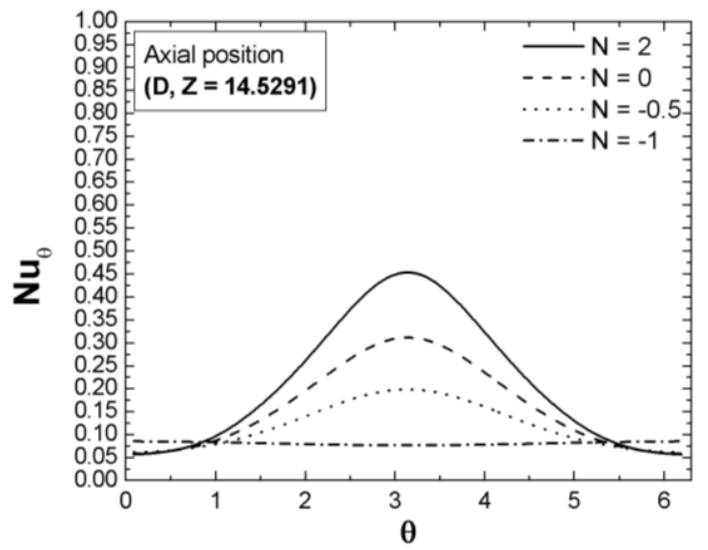

(b)

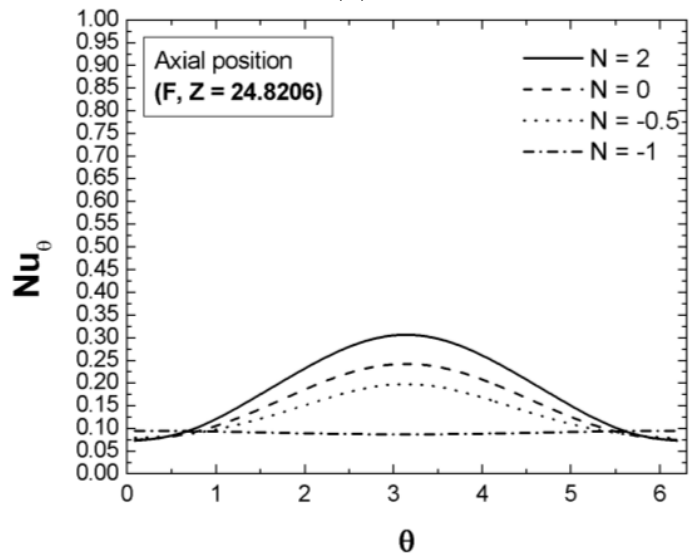

(c)

Figure 10. Effect of buoyancy ratio $N$ on the local Nusselt number at different positions $\mathrm{A}, \mathrm{D}$ and $\mathrm{F}$

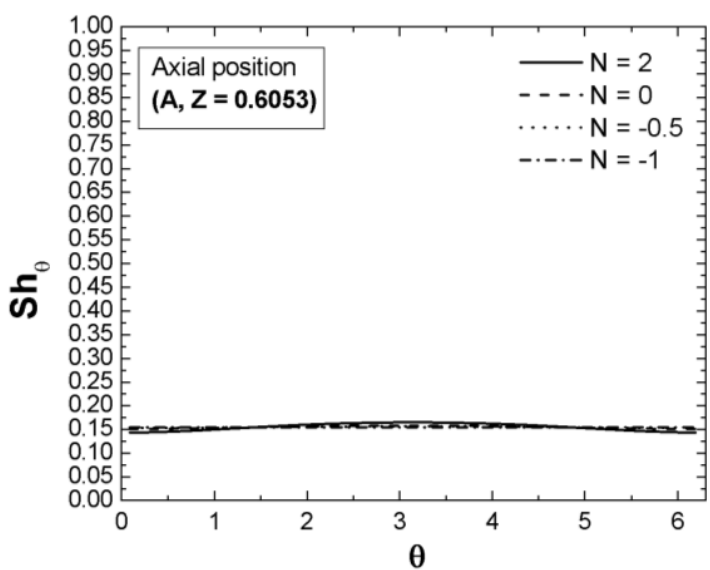

(a)

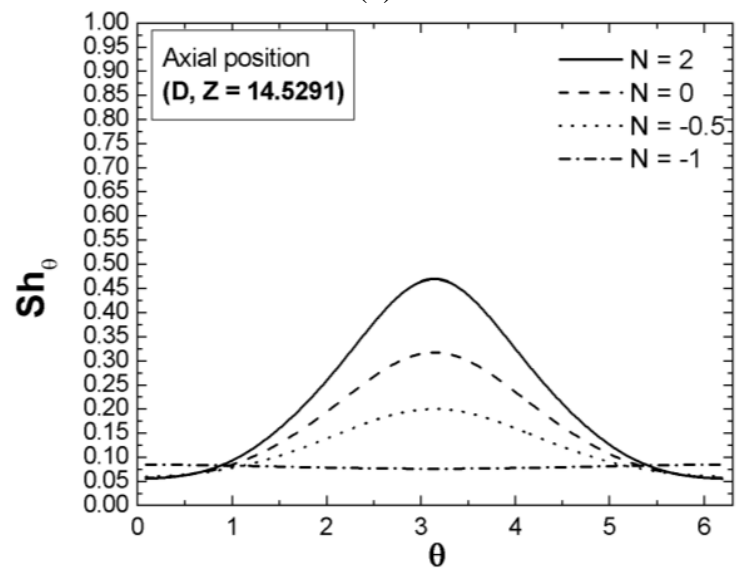

(b)

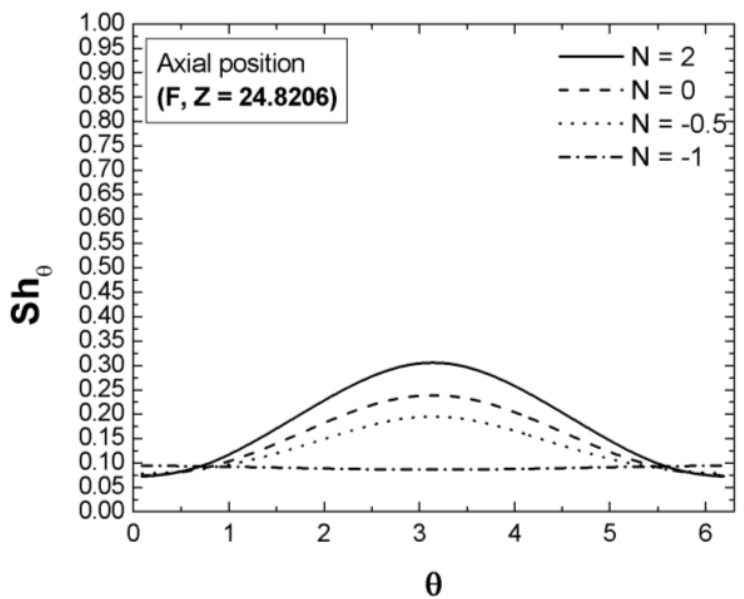

(c)

Figure 11. Effect of buoyancy ratio $N$ on the local Sherwood number at different positions $\mathrm{A}, \mathrm{D}$ and $\mathrm{F}$

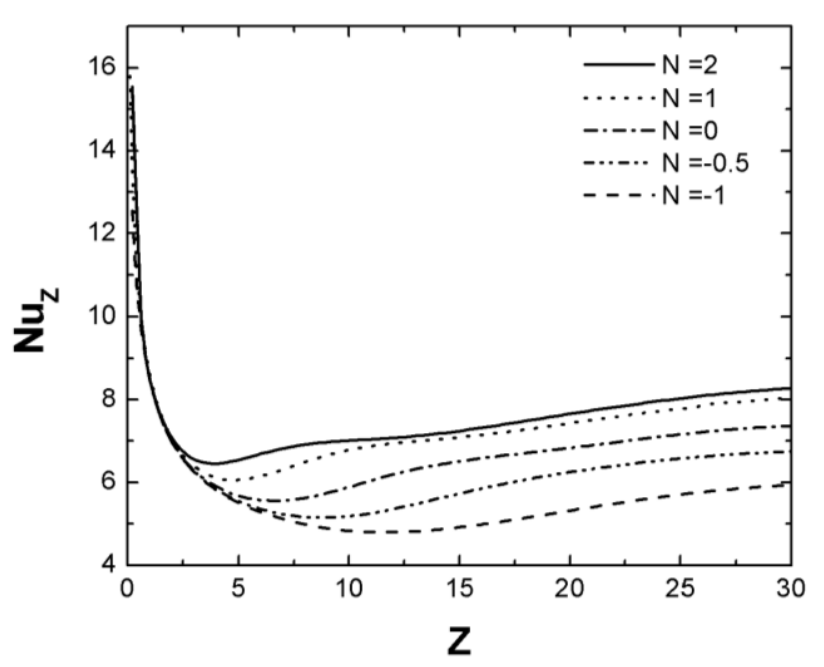

Figure 12. Effect of buoyancy ratio $N$ on the circumferentially averaged Nusselt number

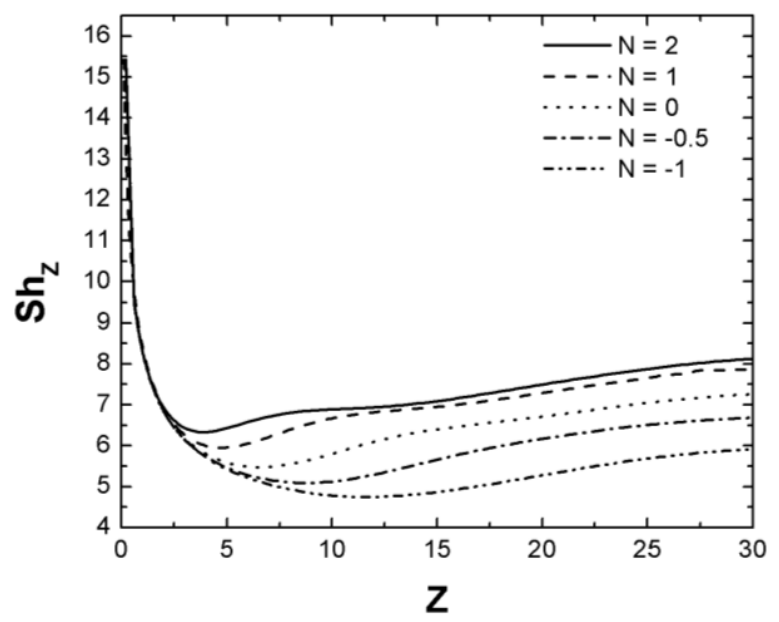

Figure 13. Effect of buoyancy ratio $N$ on the circumferentially averaged Sherwood number

The effect of the buoyancy ratio $N$ on the axial evolution of the circumferentially averaged Nusselt and Sherwood numbers characterizing the heat and mass transfer are illustrated in Figs. 12 and 13. These figures clearly show that the values of $N u_{z}$ and $S h_{z}$ close to the entrance of the middle 
part of the tube are identical, i.e. the influence of the buoyancy ratio $N$ is negligible. Further downstream, the curves of $N u_{Z}$ and $S h_{Z}$ are influenced by buoyancy ratio $N$. Thus, it is noted that these numbers increase when the solutal buoyancy force acts in the same direction as the thermal buoyancy forces (i.e. $N>0$ ). In the case of $N<0$ (the solutal buoyancy force acts in the opposite direction of the thermal buoyancy force), the numbers of $N u_{Z}$ and $S h_{Z}$ decrease with the increase in buoyancy ratio $N$. Similar results were obtained by Yan (1994) for a rectangular duct using parabolic model.

\section{CONCLUSIONS}

In this work, combined heat and mass transfer in mixed convection in a horizontal tube with uniform heat and mass fluxes on a part of its length was studied numerically. The three-dimensional elliptic form of the conservation equations was used. The effects of the Schmidt number and buoyancy ratio $N$ on the fluid flow and heat and mass transfer are examined. The principal results are as follows:

1) Near the entrance of the middle part of the tube where a constant heat and mass flux values are prescribed, the effects of the Schmidt number $S c$ and buoyancy ratio $N$ are negligible. 2) As the fluid moves downstream, the secondary flow appears because of the effect of thermal and solutal buoyancy forces, and the intensity of these secondary movements depends on a Schmidt number $S c$ and on the buoyancy ratio $N$. In addition, the velocity profiles are highly distorted by the solutal buoyancy force when $N$ is positive.

3) The increase in the Schmidt number $S c$ improves the rate of mass transfer.

4) Smaller $S c$ increases the rate of heat transfer.

5) The rates of heat and mass transfer increase when $N$ is positive and decreases when $N$ is negative.

\section{REFERENCES}

[1] Bouhezza A, Kholai O, Boudebous S, Nemouchi Z. (2013). Mixed convection heat and mass transfer in inclined circular ducts. Heat Transfer Research 44(2): 163-193.

[2] Chang CJ, Lin TF, Yan WM. (1986). Natural convection flows in a vertical open tube resulting from combined buoyancy effects of thermal and mass diffusion. Int. J. Heat Mass Transfer 29: 1543-1552.

[3] Choi DK, Choi DH. (1994). Developing mixed convection flow in a horizontal tube under circumferentially non-uniform heating. Int. J. Heat Mass Transfer 37: 1899-4913.

[4] Choudhury D, Patankar SV. (1988). Combined forced and free laminar convection in the entrance region of an inclined isothermal tube. J. Heat Transfer, ASME 110: 901-909.

[5] Gebhart B, Pera L. (1971). The nature of vertical natural convection flows resulting from the combined buoyancy effects of thermal and mass diffusion. Int. J. Heat Mass Transfer 14: 2025-2050.

[6] Joye DD. (1996). Comparison of aiding and opposing mixed convection heat transfer in a vertical tube with Grashof number variation. Int. J. Heat Fluid Flow 17(2): 96-101.

[7] Kakac S, Shah RK, Aung W. (1987). Handbook of Single-Phase Convective Heat Transfer, New York, Wiley.

[8] Kays MW, Crawford ME. (1993). Convective heat and mass transfer. Series in Mechanical Engineering, McGraw-Hill, 601.

[9] Lee KT. (1999). Laminar natural convection heat and mass transfer in vertical rectangular ducts. Int. J. Heat Mass Transfer 42: 4523-4534.

[10] Maré T, Galanis N, Voicu I, Miriel J. (2006). Experimental analysis of mixed convection in inclined tubes. App. Therm. Eng. 26: 1677-1683.

[11] Mohammed HA. (2008). Laminar mixed convection heat transfer in a vertical tube under buoyancy-assisted and opposed flows. Energy Conversion and Management 49: 2006-2015.

[12] Patankar SV. (1980). Numerical heat transfer and fluid flow. Hemisphere Publishing, New York.

[13] Orfi J, Galanis N. (2005). Mixed convection with heat and mass transfer in horizontal tubes. Int. Comm. Heat Mass Transfer 32: 511-519.

[14] Yan WM. (1995). Transport phenomena of developing laminar mixed convection heat and mass transfer in inclined rectangular ducts. Int. J. Heat Mass Transfer 38: 2905-2914.

[15] Zghal M, Galanis N, Nguyen CT. (2001). Developing mixed convection with aiding buoyancy in vertical tubes: A numerical investigation of different flow regimes. Int. J. Therm. Sci. 40: 816-824.

\section{NOMENCLATURE}

$a$

C

$C^{*}$

$C_{p}$

$D$

$g$

$G r_{C}$

$G r_{T}$

$k$

$L_{1}, L_{3}$

$L_{2}$

$\mathrm{Nu} u_{Z}$

$P^{*}$

$P$

$P r$

$q_{w}$

$r$

$R$

Re

$R i_{C}$

$R i_{T}$

$S c$

$S h_{Z}$

$T^{*}$

$T$

$t$

$u$

$u^{*}$ thermal diffusivity, $\mathrm{m}^{2} \cdot \mathrm{s}^{-1}$

dimensionless species concentration

dimensional species concentration, $(\mathrm{kg}$ of species $\mathrm{B} / \mathrm{kg}$ of mixture $(\mathrm{A}+\mathrm{B})$ )

specific heat of the fluid, $\mathrm{J} \mathrm{kg}^{-1} \cdot \mathrm{K}^{-1}$ mass diffusivity, $\mathrm{m}^{2} \cdot \mathrm{s}^{-1}$

gravitational acceleration, $\mathrm{m} \cdot \mathrm{s}^{-2}$ solutal Grashof number thermal Grashof number thermal conductivity, $\mathrm{W} \cdot \mathrm{m}^{-1} \cdot \mathrm{K}^{-1}$ lengths of adiabatic and impermeable zones, $\mathrm{m}$

length of heated zone, $\mathrm{m}$ average Nusselt number

fluid pressure, $\mathrm{Pa}$

dimensionless pressure

Prandtl number heat flux imposed at the walls, W.m ${ }^{-2}$

dimensionless radial coordinate

duct radius, $m$

Reynolds number

solutal Richardson number

thermal Richardson number

Schmidt number

average Sherwood number

fluid temperature, $\mathrm{K}$

dimensionless temperature

dimensionless time

dimensionless radial velocity

fluid radial velocity, $\mathrm{m} . \mathrm{s}^{-1}$

dimensionless axial velocity 
$v^{*} \quad$ fluid axial velocity, $\mathrm{m} \cdot \mathrm{s}^{-1}$

$w \quad$ dimensionless circumferential velocity

$w^{*} \quad$ fluid circumferential velocity, $\mathrm{m} . \mathrm{s}^{-1}$

$z$

\section{Greek symbols}

$\beta_{T}$

coefficient of thermal expansion, $\mathrm{K}^{-1}$

$\beta_{C} \quad$ coefficient of concentration expansion

$\theta \quad$ circumferential coordinate

$v \quad$ kinematic viscosity, $\mathrm{m}^{2} \cdot \mathrm{s}^{-1}$

$\rho \quad$ density, $\mathrm{kg} \cdot \mathrm{m}^{-3}$

\section{Subscripts}

b bulk fluid quantity

$0 \quad$ inlet conditions

$r, \theta, z \quad$ radial, circumferential and axial directions, respectively value at the wall

\section{Superscripts}

dimensional quantity average value 